\title{
Caveolin-1, caveolin-3 and VEGF expression in the masticatory muscles of $\mathrm{mdx}$ mice
}

\section{Christiane Kunert-Keil ${ }^{1}$, Tomasz Gredes ${ }^{1}$, Silke Lucke ${ }^{1}$, Sven Morgenstern ${ }^{1}$, Agnieszka Mielczarek ${ }^{2}$, Katarzyna Sporniak-Tutak ${ }^{3}$, Tomasz Gedrange ${ }^{1}$, Alexander Spassov ${ }^{1}$}

\author{
${ }^{1}$ Department of Orthodontics, Preventive and Pediatric Dentistry, Ernst Moritz Arndt \\ University of Greifswald, Germany \\ ${ }^{2}$ Department of Conservative Dentistry, Medical University of Warsaw, Poland \\ ${ }^{3}$ Department of Dental Surgery, Pomeranian Medical University of Szczecin, Poland
}

\begin{abstract}
Duchenne muscular dystrophy (DMD) and murine X-linked muscular dystrophy (mdx), its murine model, are characterized by muscle damage and muscle weakness associated with inflammation and new vessel formation. Caveolins, dystrophin-associated proteins, are involved in the pathogenesis of DMD, because increased numbers of caveolae are found in DMD and mdx hindlimb muscles. Caveolae influence angiogenesis due to their content of vascular endothelial growth factor (VEGF) receptors. Orofacial muscles in mdx mice undergo muscle necrosis followed by muscle regeneration. To ascertain the role of caveolins and VEGF in the pathogenesis of dystrophic masticatory muscles, we examined the expression of caveolin-1 (cav-1), caveolin-3 (cav-3) and VEGF in control and mdx mice. In mdx masticatory muscles, no changes in transcript and protein levels of VEGF were found, whereas cav-1 and cav-3 expression was increased. Using immunohistochemistry, a strong sarcolemmal staining of caveolin-3 in regenerated muscle fibers was found. Furthermore, immunohistochemistry with the caveolin-1 antibody showed an increase in the amount of blood vessels in areas with regenerating muscle fibers. Dystrophic masticatory muscles showed changes comparable to those of hindlimb muscles in the expression of cav-1 and cav-3. The angiogenesis seems to be unaffected in the jaw muscles of mdx mice. We speculate that the increased caveolin expression could cause extensive and efficient muscle regeneration. (Folia Histochemica et Cytobiologica 2011; Vol. 49, No. 2, pp. 291-298)
\end{abstract}

Key words: mdx mice, muscular dystrophy, histopathology, masticatory muscles

\section{Introduction}

Caveolae are vesicular invaginations of the plasma membrane and transport molecules across the endothelial barrier. Caveolae are also involved in phagocytosis, signal transduction regulation and cholesterol transport [1]. Caveolins, a gene family of $21-25 \mathrm{kDa}$ integral membrane proteins, are the main protein components of caveolae membranes. The mammali-

\footnotetext{
Correspondence address: Ch. Kunert-Keil, Department of Orthodontics, Preventive and Pediatric Dentistry, Ernst Moritz Arndt, University of Greifswald, Rotgerberstr. 8, D-17475 Greifswald, Germany; tel.: (+ 49 3834) 8671 56, fax: (+ 49 3834) 8671 13; e-mail: keil@uni-greifswald.de
}

an caveolin gene family consists of three different isoforms: caveolin1-3 [1]. Caveolin-1 and caveolin-2 are usually expressed in the same cell type, like adipocytes, endothelial cells and type I pneumocytes [2]. Caveolin-1 is able to bind several proteins via strongly hydrophobic scaffolding domains [3] and regulates important functions such as angiogenesis, vascular permeability and transcytosis [4, 5]. In a recent study on caveolin-deficient mice, angiogenesis was found to be markedly reduced in comparison with control mice [6]. Caveolin-3 (cav-3) is present in all muscle cell types and is the major caveolar protein of differentiated skeletal muscle cells, but it is also expressed in astrocytes and chondrocytes [7-9]. In skeletal muscle, cav-3 is transiently associated with T-tubules during development [10]. Immunohistochemistry of cav-3 
in muscle fibers reveals a co-localization of this protein with dystrophin at the sarcolemma [11]. Furthermore, cav-3 defects have been closely tied to muscular dystrophy [12]. Mutation in the cav-3 gene causes autosomal dominant muscle diseases $[13,14]$. Transgenic overexpression of cav-3 has been found to induce a phenotype similar to Duchenne muscular dystrophy (DMD) [15].

Murine X-linked muscular dystrophy ( $\mathrm{mdx}$ ) and Duchenne muscular dystrophy (DMD) are characterized by a complete lack of dystrophin [16, 17]. Dystrophin deficiency leads to necrosis of muscle fibers, muscle damage, fibrosis and progressive muscle weakness [18]. Recent studies have identified a characteristic increase in the number of caveolae in DMD patients and mdx mice [19-21] and an increase in the expression of cav-3 levels in DMD and mdx hindlimb muscles [21, 22].

Beside caveolins, caveolae contain receptors for key regulators of vascular permeability, the vascular endothelial growth factors (VEGFs). VEGFA activates vascular permeability, vasodilatation and new vessel formation [23]. VEGF can be generated by almost all cells under hypoxic conditions and are highly expressed in tissues undergoing growth [24]. Recently it has been shown that cav-1 expression correlates positively with VEGF expression [25] and cav-1 plays multiple roles in the VEGF-induced signaling cascade [26]. Because of the interactions of caveolins with VEGF, changes in the abundance of caveolins are likely to influence inflammation and angiogenic processes in the dystrophic muscles of mdx mice. Caveolins have been intensively investigated in smooth and limb skeletal muscles, but until now little attention has been paid to their abundance in muscles of the head and neck region. However, orofacial muscles are constantly loaded under important physiological conditions such as eating, swallowing and breathing, and muscle diseases may seriously impact these functions.

Recently, it has been shown that the masticatory muscles of mdx mice are characterized by typical cycles of necrosis and regeneration associated with inflammation, muscle fiber hypertrophy and collagen accumulation [27].

The objective of this study was therefore to analyze the expression of cav-1, cav-3 and VEGF in the tongue, masseter and temporal muscles, and one limb skeletal muscle in 100 day-old mdx mice in order to examine their potential roles in the regeneration of masticatory muscles. At this age, in mdx mice, the regeneration process overwhelms degeneration. Literature is scarce regarding the caveolin expression within newly regenerated fibers. Our results could provide a framework for investigating the pathogenesis of DMD in the mdx masticatory system and its impact on the craniofacial bone. The results of the presented study will help demonstrate how masticatory muscles adapt to dystrophic changes.

\section{Material and methods}

Animals. Mice of the inbred strains C57Bl/10ScSn (control) and C57/Bl10ScSn-Dmd ${ }^{m d x} / \mathrm{J}(\mathrm{mdx})$ were originally obtained from Harlan Winkelmann (Borchen, Germany) and Charles River (Sulzfeld, Germany). Both strains were bred in the Department of Pathophysiology of the Medical Faculty at the University of Greifswald. Age-matched pairs of mdx and control animals (each $n=9-10 ; 100$ days old) of either sex were euthanized using ether. All procedures were approved by a governmental committee on animal welfare of the State Government (LALLF M-V/TSD/7221.3-2.3-001/09). Tongue, masseter, temporalis and soleus muscles were prepared and shock-frozen in liquid nitrogen for quantitative reverse transcription PCR, Western blot analyses and immunohistochemistry.

RNA extraction and reverse transcription. Total RNA was isolated using guanidinium-isothiocyanate (RNeasy Fibrous Mini Kit, Qiagen, Hilden, Germany) and RNA concentration was determined by UV absorbance measurements. An amount of 200 ng total RNA was reverse transcribed using random hexamer primers and the TaqMan Reverse Transcription Reagents (PE Applied Biosystems, Weiterstadt, Germany), as described previously [28, 29].

TaqMan RT-PCR. The method was performed as described previously $[30,31]$ using a real-time PCR cycler (StepOne Plus, Applied Biosystems). Gene-specific primers and probes were purchased from PE Applied Biosystems (cav-1: Mm00483057_m1; cav-3: Mm01182632_m1; VEGF: Mm01281449_m1) with each probe having been synthesized with a fluorescent 5'-reporter dye (FAM: 6-carboxy-fluorescein) and a 3'-quencher dye (TAMRA: 6-carboxy-tetramethyl-rhodamine). All values are given in relation to the mRNA of 18S rRNA. A 'no-template control' with water was performed parallel to all experiments. Each series of experiments was performed twice.

Western blot analysis. Muscle tissue samples were mechanically homogenized using the SpeedMill P12 homogenizer (Analytikjena, Jena, Germany). Total protein extracts $(30 \mu \mathrm{g})$ were separated on SDS gels, transferred to nitrocellulose membranes (Schleicher\&Schuell) using a tank blotting system (Gibco-BRL, Germany) and incubated with antibodies against caveolin-1 (polyclonal, Affinity Bioreagents, via Dianova, Hamburg, Germany, dilution 1:1,000 in PBS containing $5 \%$ powdered milk and $0.025 \% \mathrm{NaN}_{3}$ ) or caveolin-3 (monoclonal, BD Biosciences, Heidelberg, Germany, dilution 1:400 in PBS containing 5\% powdered milk and $0.025 \%$ 
$\mathrm{NaN}_{3}$ ) overnight at $4^{\circ} \mathrm{C}$. Secondary HRP-conjugated goat anti-rabbit or goat anti-mouse immunoglobulins (Dako, Hamburg, Germany) were used at a dilution of 1:5,000. Visualization and detection of bound antibodies were carried out using an enhanced chemiluminescence system (Perbio Science). As control for the specificity of the antibodies, antigen-preincubated antibodies $(1 \mu \mathrm{g}$ peptide per $1 \mu \mathrm{g}$ of antibody) were used in the same way. To assess equal loading of the gel, every membrane was stripped with Restore ${ }^{\mathrm{TM}}$ Plus Western blot stripping buffer (Perbio Science) and incubated with a monoclonal anti- $\alpha$-actinin antibody (clone AT6/172, Upstate, dilution 1:1,000; two hours at room temperature). Quantitative analyses of protein bands from cav-1, cav-3 and $\alpha$-actinin in masticatory muscles were carried out using GelScan 5.2 software (Serva, Germany). MOD (mean optical density) \pm SEM are given in all cases for $n=4$ muscle samples and four independent Western blot analyses.

Immunohistochemistry. Indirect immunohistochemistry was performed on acetone or methanol/ $\mathrm{H}_{2} \mathrm{O}_{2}$ fixed cryo-sections ( $5 \mu \mathrm{m} ; \mathrm{n}=4$ different animals) using a polyclonal anti-caveolin-1 antibody (Affinity Bioreagents via Dianova, Hamburg, Germany, dilution 1:1,000) or a monoclonal mouse anti-caveolin-3 antibody (clone 26, BD Biosciences, Heidelberg, Germany, dilution 1:400), as described previously [32]. Staining was performed following the instructions for the $\mathrm{ABC}$-systems (Vectastain ABC kit, Vector Laboratories, Burlingame, CA, USA) either with a biotinylated secondary anti-rabbit antibody or the M.O.M.-kit (Vector Laboratories). Incubation with the primary antibodies was done for one hour at room temperature. Visualization of bound cav-1 and cav-3 antibodies was done using a New Fuchsine alkaline phosphatase substrate protocol. Sections were counterstained in hematoxylin and then cover-slipped. In negative controls, the primary antibody was replaced by PBS, and PBS used in the same way.

A simultaneous blind test was conducted using identical staff, equipment and chemicals to determine the level of antigen. From each section, five digital pictures were taken, at random, of different parts of the tissue $(200 \times$ magnification; 3CCD color camera; Hitachi HV-C20M; Hitachi Denshi Ltd, Japan, and Axiolab, Carl Zeiss, Göttingen, Germany). For standardization of the measurement in each picture, the optical density of white background color was attuned to 250. For all sections, we assessed the MOD and the quantity of pixels that had a positive reaction for cav-1 and cav-3 using KSRun software (imaging system KS400, release 3.0; Zeiss, Vision $\mathrm{GmbH}$, Munich, Germany) or ImageJ 1.42 for Windows (http://rsbweb.nih.gov/ij/).

Statistical analysis. All statistical analyses were performed using SigmaPlot software (Systat Software, Inc., San Jose, CA, USA). Statistical analyses were made using Student's unpaired $t$-test. Data is given as means \pm SEM; $\mathrm{p}<0.05$ was considered statistically significant.

\section{Results}

\section{Expression of cav-1, cav-3 and VEGF in masticatory muscles of $\mathrm{mdx}$ mice}

Quantitative TaqMan RT-PCR performed on masticatory muscle tissue samples and soleus muscle revealed the presence of cav-1, cav-3 and VEGF in $\mathrm{mdx}$ and control mice. Since we observed no differences in gene expression between male and female animals, the data from both sexes was combined.

The mRNA expression of cav-1 was 2.8-fold increased in dystrophic masseter muscle compared to controls (control vs. mdx: $39 \pm 4$ vs. $110 \pm 19 ; * * \mathrm{p}=$ $=0.0103)$. The mRNA expression of cav-3 and VEGF remained unchanged in mdx masseter muscle (Figure 1). In temporal and tongue muscles, no significant differences were observed for all three tested transcripts between control and mdx mice. In contrast, the soleus muscle from mdx mice showed significantly lower transcript levels of VEGF (control vs. mdx: $571 \pm 94$ vs. $291 \pm 45 ; * \mathrm{p}=0.0234$ ). The VEGF mRNA level reached about 70-80\% compared to those in non-dystrophic control mice (Figure 1), while cav-3 expression significantly increased (1.5-fold; control vs. mdx: $218 \pm 13$ vs. $327 \pm$ $\left.\pm 59 ;{ }^{*} \mathrm{p}=0.0496\right)$ and cav-1 expression remained unchanged.

Western blot analysis allowed us to semi-quantify cav-1 and cav-3 proteins (Figure 2). Single immunoreactive bands of approximately 22 and $18 \mathrm{kDa}$, respectively, were detected. These bands agreed with the expected molecular weights of cav1 and cav-3 (Figure 2A). Using antigen pre-incubated antibodies, no specific immunoreactive bands were detected. Quantitative evaluation of the Western blots showed slightly increased levels of cav-1 protein in all tested muscles of mdx mice (mean \pm SEM; control vs. mdx: masseter, $1.24 \pm 0.25$ vs. $1.21 \pm 0.12$; temporalis, $1.38 \pm 0.19$ vs. $1.46 \pm 0.17$; tongue, $0.74 \pm$ \pm 0.18 vs. $0.89 \pm 0.18$; soleus, $0.82 \pm 0.18$ vs. $0.95 \pm$ \pm 0.21 ). The protein levels were 106-119\% of those of control mice (Figure 2B). Quantitative evaluation of cav-3 Western blots showed a tendency towards increased levels between $\mathrm{mdx}$ and control mice in tongue, temporal and soleus muscle (mean $\pm \mathrm{SEM}$; control vs. mdx: temporalis, $1.0 \pm 0.35$ vs. $1.51 \pm 0.37$; tongue, $1.13 \pm 0.38$ vs. $1.49 \pm 0.28$; soleus, $1.08 \pm$ \pm 0.02 vs. $1.83 \pm 0.22$ ). A 1.3 - to 1.7 -fold increase in protein expression in dystrophic muscles was detected (Figure 2C). Only in mdx masseter muscle were reduced protein levels detected compared to control muscle (mean \pm SEM; control vs. mdx: $1.69 \pm 0.23$ vs. $1.37 \pm 0.41)$. 
$\mathbf{A}$
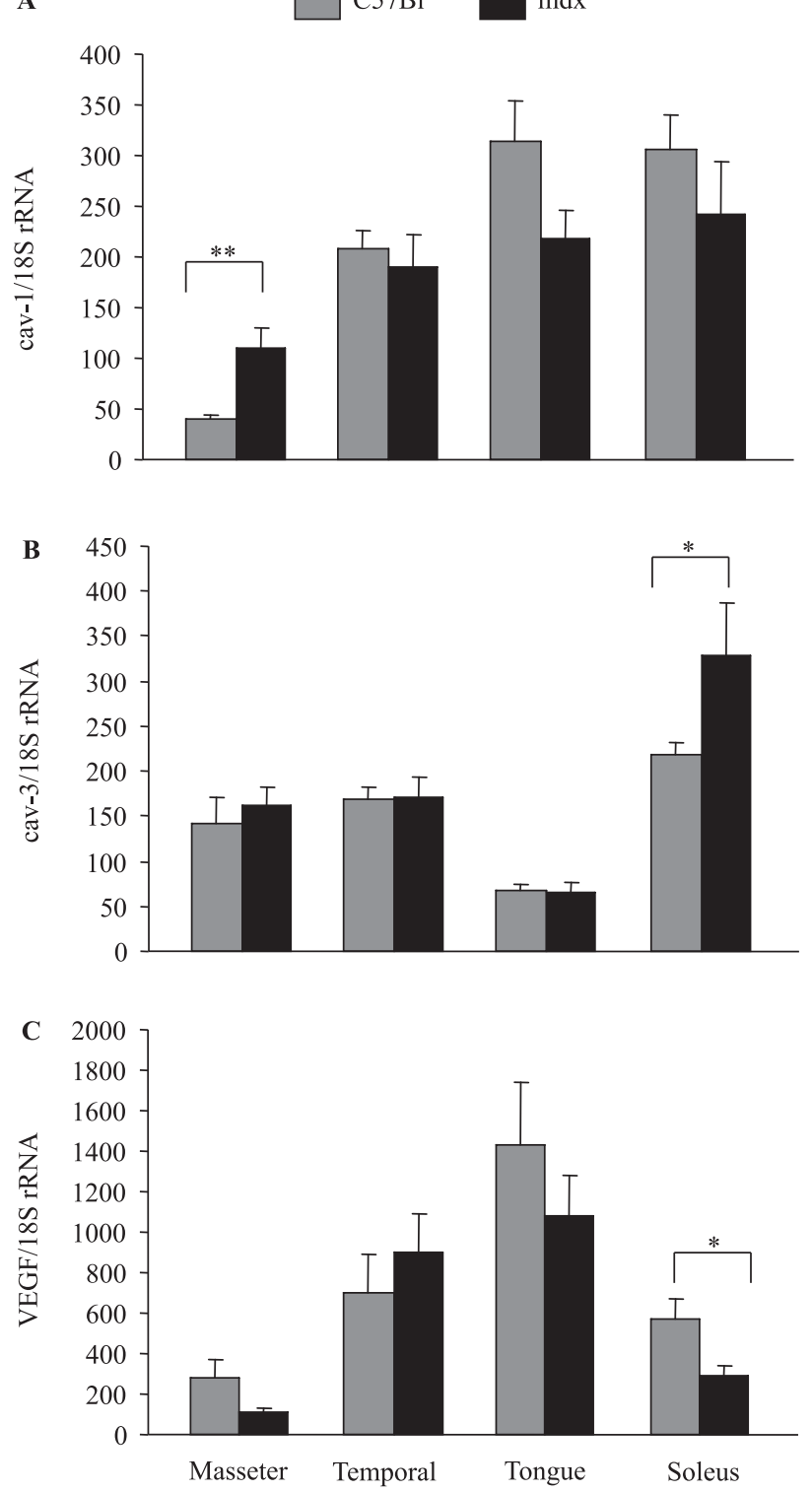

Figure 1. Quantification of cav-1, cav-3 and VEGF mRNA levels in 100 day old control (grey columns) and mdx (black columns) mice. Levels of mRNA were determined using RNA preparations from masseter, temporal and soleus muscles as well as the tongue. The mRNA levels of all tested genes are given in relation to that of $18 \mathrm{~S}$ rRNA. Means \pm SEM are given in all cases for $\mathrm{n}=9-10$ samples. Stars indicate significant differences: ${ }^{*} \mathrm{p}<0.05$; ${ }^{* *} \mathrm{p}<0.01$, unpaired $t$-test

\section{Cellular localization of cav-1 and cav-3 in masticatory muscles}

Using immunohistochemistry with the polyclonal antibody against cav-1, we found a strong staining of endothelial and satellite cells in muscle cross-sections of both mouse strains (Figure 3A). Computerized analysis of the staining intensity (optical density) is shown in Figure 3B. We found a significant increase in cav-1 protein expression in the masseter and tem-
A
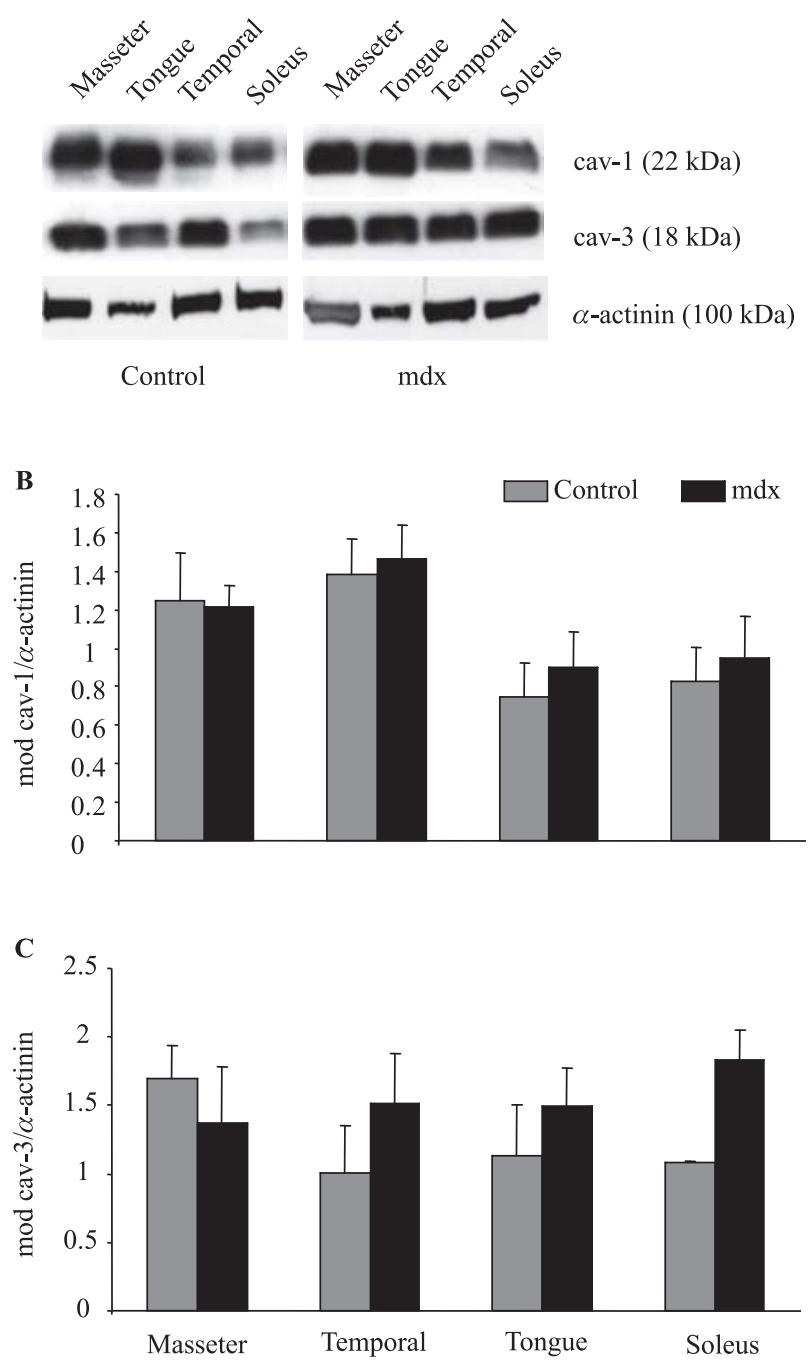

Figure 2. Detection of cav-1 and cav-3 in masticatory and soleus muscles. (A) Representative Western blots of cav-1 and cav-3 in masseter, temporal and soleus muscles as well as tongue from control and mdx mice. The membranes were probed with a polyclonal antibody against cav-1 and $\alpha$-monoclonal antibody against cav-3. A monoclonal antibody was used to detect a-actinin serving as an internal control. (B) Quantitative analyses of cav-1 Western blots as shown in A. (C) Quantitative analyses of cav-3 Western blots as shown in A. Protein bands attributed to cav-1, cav-3 and $\alpha$-actinin were evaluated using GelScan 5.2 software (Serva, Germany). Mean optical densities (MOD) \pm SEM of control and mdx mice are given in all cases for four independent experiments

poral muscles of mdx mice compared to controls (masseter: control vs. $m d x=536 \pm 64$ vs. $966 \pm 149 ; * \mathrm{p}=$ $=0.017$; temporal: control vs. $\mathrm{mdx}=420 \pm 56$ vs. $696 \pm$ $\pm 115 ; * \mathrm{p}=0.046$ ). In addition, there were no significant differences in cav-1 protein expression in tongue and soleus muscle between control and mdx mice.

Immunohistochemical staining of muscle cross-sections showed the presence of cav-3 proteins in 
A
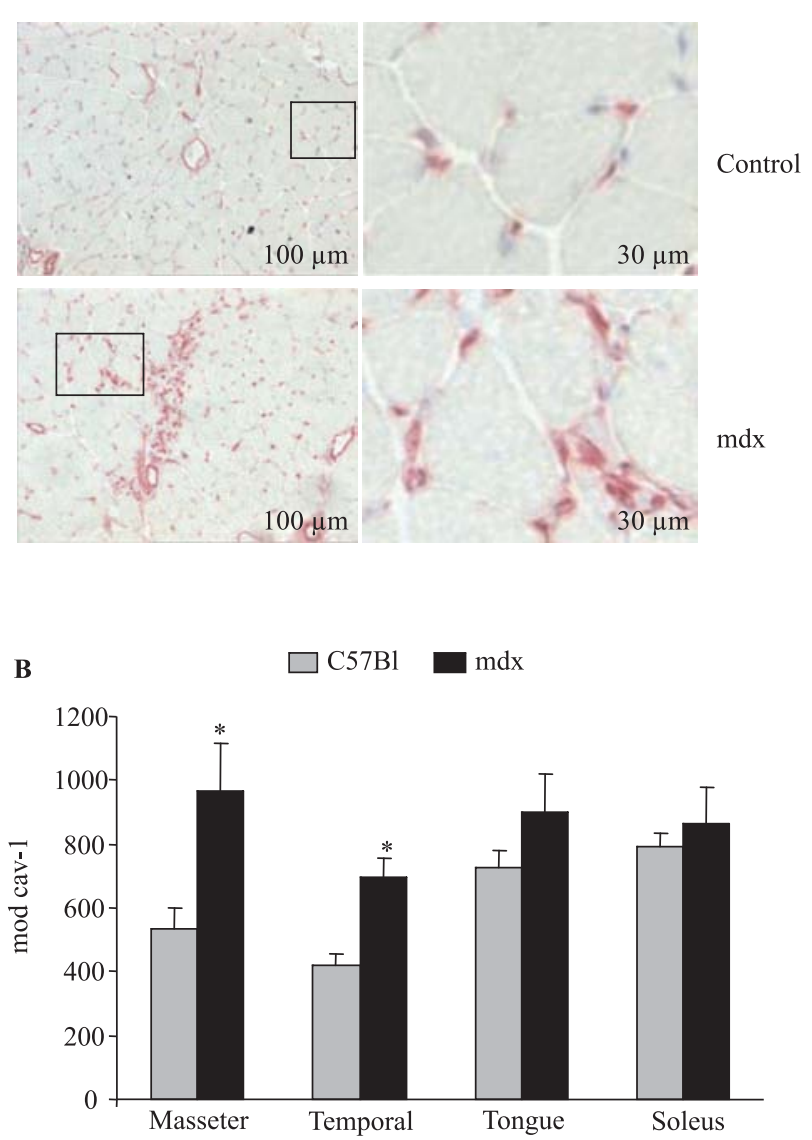

Figure 3. (A) Immunohistochemical staining of cav-1 in cryo-sections of masseter muscle samples from control and mdx mice at two different magnifications. Cav-1 staining is in red; counterstaining of the nuclei with hemalaun (blue). (B) Densitometric analysis of the cav-1 staining intensity. The mean optical densities (MOD) \pm \pm SEM of control and mdx mice are given in all cases. Stars indicate significant differences: ${ }^{*} \mathrm{p}<0.05$; *** $\mathrm{p}<$ 0.005 , unpaired $t$-test

the sarcolemma of control and mdx muscle fibers (Figure 4A). The cav-3 antigen expression determined by densitometric analyses of the immunohistochemical stained sections was 1.5 -fold increased in the masseter muscle, and 1.3-fold in the tongue and soleus muscles, of mdx mice compared to the levels of cav-3 in control mice (MOD, masseter: control vs. $\mathrm{mdx}=1,876 \pm 144$ vs. $2,876 \pm 321 ; \mathrm{p}=$ $=0.013$; tongue: control vs. $\mathrm{mdx}=2,711 \pm 165 \mathrm{vs}$. $3,520 \pm 217 ; \mathrm{p}=0.0084$; soleus: control vs. $\mathrm{mdx}=$ $=2,577 \pm 250$ vs. $3,303 \pm 189 ; \mathrm{p}=0.034)$. No significant differences in cav-3 protein expression were found in the temporal muscle of either experimental group (Figure 4B).

All sections incubated with PBS instead of the primary antibody were devoid of any positive reaction.
A

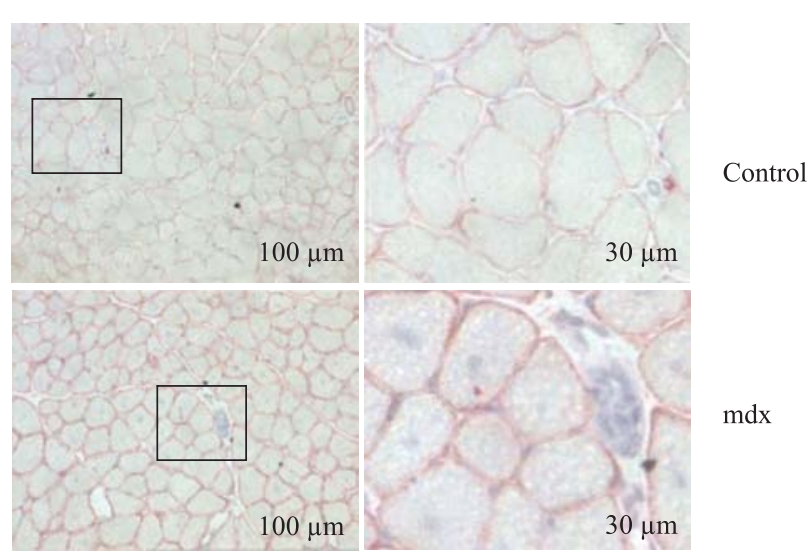

B

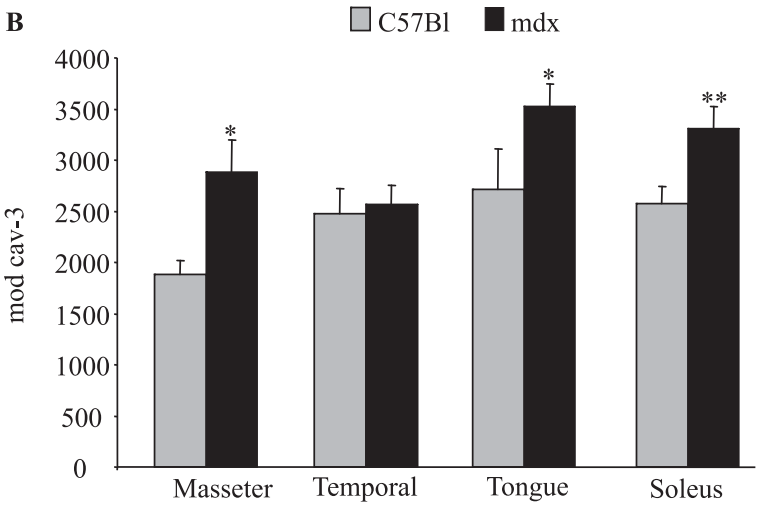

Figure 4. (A) Immunohistochemical staining of cav-3 in cryo-sections of masseter muscle samples from control and mdx mice at two different magnifications. Cav-3 staining is in red; counterstaining of the nuclei with hemalaun (blue). (B) Densitometric analysis of the cav-3 staining intensity. The mean optical densities (MOD) \pm SEM of control and mdx mice are given in all cases. Stars indicate significant differences: ${ }^{* * *} \mathrm{p}<0.005$, unpaired $t$-test

The involvement of caveolae and caveolins in the pathogenesis of Duchenne muscular dystrophy has been suggested, because: (i) cav-3 has been found to be localized to the sarcolemma, which coincides with the distribution of dystrophin and co-fractionates with members of the dystrophin complex [11]; (ii) skeletal muscle caveolae have been found to undergo characteristic changes in size and distribution in patients with DMD [19]; and (iii) transgenic overexpression of cav-3 induces a phenotype similar to DMD [15].

Furthermore, caveolae and caveolins are clearly important to vascular function, angiogenesis and during inflammation [12]. It has recently been shown that mdx orofacial muscles, e.g. masseter and temporal, were severely affected by dystrophin deficiency, while $\mathrm{mdx}$ tongue muscle was partially spared. These chang-

\section{Discussion}


es were found to be accompanied by intensive regeneration, with numerous inflammatory foci and new vessel formations [27].

In our study, we analyzed the expression of caveolin- 1 and -3 as well as VEGF in the masticatory muscles of the animal model of DMD, the mdx mice. With regard to the expression of caveolin-3, the tested masticatory muscles showed significant differences on the protein level between $\mathrm{mdx}$ and control mice. Our results on masticatory muscles do not differ from those using hindlimb muscles, because in dystrophin-negative soleus muscle, an increase in the cav-3 expression was also observed. The increased abundance of cav-3 in the masticatory muscles and soleus of 100 day old mdx mice is consistent with previous reports demonstrating that cav-3 is upregulated in $\mathrm{mdx}$ tibialis anterior [22], in mdx extensor digitorum longus [33], in the muscles of dystrophic chickens [34] and in the quadriceps of DMD patients [21].

However, the mechanisms by which cav-3 is involved in the mdx/DMD dystrophin deficiency remains unclear. New observations suggest that cav-3 expression is necessary for transient receptor potential cation channel canonical 1 (TRPC1) localization. TRPC1 and cav-3 are co-localized and co-immunoprecipitated [35]. TRPC1 is a stretch-activated $\mathrm{Ca}^{2+}$ channel and seems to play a role in $\mathrm{Ca}^{2+}$ induced membrane damage in $\mathrm{mdx} / \mathrm{DMD}$ skeletal muscle fibers [36].

It seems possible that as well as cav-3, cav- 1 could also play an important role in the pathogenesis of DMD, because cav-1 knockout mice displayed numerous skeletal muscle abnormalities, including tubular aggregates [37]. Cav-1 plays an essential role in the regulation of cell proliferation.

It has recently been shown that skeletal muscle fibers from fetuses at late gestation stage, and from newborns, express cav-1, whereas adult samples do not show any immunoreaction for caveolin-1 [38]. The expression of cav-1 in the skeletal muscle could also be a marker for muscle regeneration as well as for inflammation. In the tested dystrophic masticatory muscles, we found an increase in the expression of cav-1. Because both protein and mRNA were extracted from the whole muscle sample containing muscle fibers, blood vessels and connective tissue, it seems possible that the increased concentration of cav- 1 is due to: (i) formation of new blood vessels during regeneration: the numerous inflammatory foci found in $\mathrm{mdx}$ masticatory and soleus muscles are associated with new vessel formation and leukocyte accumulation [27]; (ii) by fibroblasts, which are responsible for increased collagen and connective tissue content in $\mathrm{mdx}$ muscles [27, 39, 40]; and (iii) by the physio- logical abundance of adipocytes. It is known that cav-1 is mainly expressed in adipocytes, smooth muscle cells, fibroblasts, and endothelial cells [5, 41, 42]. Unlike the other masticatory muscles, in the tongue we did not find changes in the level of cav- 1 . This could explain our earlier results which showed a virtually unaffected tongue muscle, with absence of inflammation and collagen accumulation [27].

Angiogenesis is necessary for muscle regeneration. The proliferation of capillary endothelial cells is stimulated by VEGF, a critical regulating factor in angiogenesis [43]. In DMD patients, increased VEGF serum levels have been detected [44]. On the other hand, mdx mice treated with recombinant VEGF have shown increased forelimb strength and regenerating fibers and reduced necrotic areas [45]. In our study, unchanged or reduced mRNA amounts of VEGF were found in the tested dystrophic muscles compared to controls.

It is worth emphasizing that we analyzed VEGF only on the transcriptional level, and our results for VEGF should be verified on the level of protein expression. However, there is clear evidence of a close relationship between VEGF and caveolins. VEGFR-2 has been found to be localized in caveolae and cav-1 has been shown to reduce VEGF-stimulated angiogenesis in vivo, demonstrating direct cav-1 regulation of angiogenesis $[12,46]$.

To sum up, in the masticatory muscles of mdx mice we found an increase in cav- 1 and cav- 3 expression, whereas the amount of VEGF seemed to be unchanged. The differences in the cav- 1 and cav- 3 expression between the studied muscles confirm previous observations that muscles in mdx mice are unequally affected by dystrophin deficiency. We concluded that dystrophic masticatory muscles of 100 day old mdx mice showed complex muscle adaptation during the muscle regeneration process.

\section{Acknowledgements}

We thank I. Pieper for excellent technical assistance.

\section{References}

1. Couet J, Belanger MM, Roussel E, Drolet MC. Cell biology of caveolae and caveolin. Adv Drug Deliv Rev. 2001; 49:223-235.

2. Razani B, Woodman SE, Lisanti MP. Caveolae: from cell biology to animal physiology. Pharmacol Rev. 2002;54:431-467.

3. Couet J, Li S, Okamoto T, Ikezu T, Lisanti MP. Identification of peptide and protein ligands for the caveolin-scaffolding domain. Implications for the interaction of caveolin with caveolae-associated proteins. J Biol Chem. 1997;272:6525-6533.

4. Griffoni C, Spisni E, Santi S et al. Knockdown of caveolin1 by antisense oligonucleotides impairs angiogenesis in vitro and in vivo. Biochem Biophys Res Commun. 2000; 276:756-761. 
5. Frank PG, Woodman SE, Park DS, Lisanti MP. Caveolin, caveolae, and endothelial cell function. Arterioscler Thromb Vasc Biol. 2003;23:1161-1168.

6. Woodman SE, Ashton AW, Schubert W et al. Caveolin-1 knockout mice show an impaired angiogenic response to exogenous stimuli. Am J Pathol. 2003;162:2059-2068.

7. Nishiyama K, Trapp BD, Ikezu T et al. Caveolin-3 upregulation activates beta-secretase-mediated cleavage of the amyloid precursor protein in Alzheimer's disease. $J$ Neurosci. 1999;19:6538-6548.

8. Okamoto T, Schlegel A, Scherer PE, Lisanti MP. Caveolins, a family of scaffolding proteins for organizing "preassembled signaling complexes" at the plasma membrane. J Biol Chem. 1998;273:5419-5422.

9. Schwab W, Galbiati F, Volonte D et al. Characterisation of caveolins from cartilage: expression of caveolin-1, -2 and -3 in chondrocytes and in alginate cell culture of the rat tibia. Histochem Cell Biol. 1999;112:41-49.

10. Parton RG, Way M, Zorzi N, Stang E. Caveolin-3 associates with developing T-tubules during muscle differentiation. JCell Biol. 1997;136:137-154.

11. Song KS, Scherer PE, Tang Z et al. Expression of caveolin-3 in skeletal, cardiac, and smooth muscle cells. Caveolin-3 is a component of the sarcolemma and co-fractionates with dystrophin and dystrophin-associated glycoproteins. J Biol Chem. 1996;271:15160-15165.

12. Chidlow JH Jr, Sessa WC. Caveolae, caveolins, and cavins: complex control of cellular signalling and inflammation. Cardiovasc Res. 2010;86:219-225.

13. Minetti C, Sotgia F, Bruno C et al. Mutations in the caveolin3 gene cause autosomal dominant limb-girdle muscular dystrophy. Nat Genet. 1998;18:365-368.

14. Woodman SE, Sotgia F, Galbiati F, Minetti C, Lisanti MP. Caveolinopathies: mutations in caveolin- 3 cause four distinct autosomal dominant muscle diseases. Neurology. 2004; 62:538-543.

15. Galbiati F, Volonte D, Chu JB et al. Transgenic overexpression of caveolin-3 in skeletal muscle fibers induces a Duchenne-like muscular dystrophy phenotype. Proc Natl Acad Sci USA. 2000;97:9689-9694.

16. Hoffman EP, Brown RH Jr, Kunkel LM. Dystrophin: the protein product of the Duchenne muscular dystrophy locus. Cell. 1987;51:919-928.

17. Campbell KP. Three muscular dystrophies: loss of cytoskeleton-extracellular matrix linkage. Cell. 1995;80:675-679.

18. Emery AEH, Emery MHL. Refining the clinical picture. In: Emery AEH EM (eds.). The history of a genetic disease: Duchenne muscular dystrophy or Meryons's disease. London: The Royal Society of Medicine Press Limited 1995;89-99.

19. Bonilla E, Fischbeck K, Schotland DL. Freeze-fracture studies of muscle caveolae in human muscular dystrophy. Am J Pathol. 1981;104:167-173.

20. Ito H, Yoshimura T, Tsujihata M, Nagataki S. Distribution of intramembranous particle size in the muscle plasma membrane of the mdx mouse. J Neurol Sci. 1997;148:147-151.

21. Repetto S, Bado M, Broda P et al. Increased number of caveolae and caveolin-3 overexpression in Duchenne muscular dystrophy. Biochem Biophys Res Commun. 1999;261:547-550.

22. Vaghy PL, Fang J, Wu W, Vaghy LP. Increased caveolin-3 levels in mdx mouse muscles. FEBS Lett. 1998;431:125-127.

23. Ferrara N, Davis-Smyth T. The biology of vascular endothelial growth factor. Endocr Rev. 1997;18:4-25.

24. Bates DO. Vascular endothelial growth factors and vascular permeability. Cardiovasc Res. 2010;87:262-271.
25. Zhang ZB, Cai L, Zheng SG, Xiong Y, Dong JH. Overexpression of caveolin-1 in hepatocellular carcinoma with metastasis and worse prognosis: correlation with Vascular Endothelial Growth Factor, microvessel density and unpaired artery. Pathol Oncol Res. 2009;15(3):495-502.

26. Labrecque L, Royal I, Surprenant DS et al. Regulation of vascular endothelial growth factor receptor- 2 activity by caveolin-1 and plasma membrane cholesterol. Mol Biol Cell. 2003;14:334-347.

27. Spassov A, Gredes T, Gedrange T et al. Histological changes in masticatory muscles of mdx mice. Arch Oral Biol. 2010;55:318-324.

28. Kruger J, Kunert-Keil C, Bisping F, Brinkmeier H. Transient receptor potential cation channels in normal and dystrophic mdx muscle. Neuromuscul Disord. 2008;18:501-513.

29. Kunert-Keil C, Bisping F, Kruger J, Brinkmeier H. Tissue-specific expression of TRP channel genes in the mouse and its variation in three different mouse strains. BMC Genomics. 2006; 7:159.

30. Gredes T, Kunert-Keil C, Dominiak M et al. The influence of biocomposites containing genetically modified flax fibers on gene expression in rat skeletal muscle. Biomed Tech (Berl). 2010;55(6):331-334.

31. Gredes T, Spassov A, Mai R et al. Changes in insulin like growth factors, myostatin and vascular endothelial growth factor in rat musculus latissimus dorsi by poly-3-hydroxybutyrate implants. J Physiol Pharmacol. 2009;60(Suppl 3): $77-81$.

32. Gedrange T, Walter B, Tetzlaff I et al. Regional alterations in fiber type distribution, capillary density, and blood flow after lower jaw sagittal advancement in pig masticatory muscles. J Dent Res. 2003;82:570-574.

33. Shibuya S, Wakayama Y, Inoue M, Oniki H, Kominami E. Changes in the distribution and density of caveolin 3 molecules at the plasma membrane of mdx mouse skeletal muscles: a fracture-label electron microscopic study. Neurosci Lett. 2002;325:171-174.

34. Matsumoto H, Sasazaki S, Fujiwara A et al. Accumulation of caveolin-3 protein is limited in damaged muscle in chicken muscular dystrophy. Comp Biochem Physiol A Mol Integr Physiol. 2010;157:68-72.

35. Gervasio OL, Whitehead NP, Yeung EW, Phillips WD, Allen DG. TRPC1 binds to caveolin-3 and is regulated by Src kinase - role in Duchenne muscular dystrophy. J Cell Sci. 2008;121:2246-2255.

36. Allen DG, Gervasio OL, Yeung EW, Whitehead NP. Calcium and the damage pathways in muscular dystrophy. Can J Physiol Pharmacol. 2010;88:83-91.

37. Schubert W, Sotgia F, Cohen AW et al. Caveolin-1(-/-)- and caveolin-2(-/-)-deficient mice both display numerous skeletal muscle abnormalities, with tubular aggregate formation. Am J Pathol. 2007;170:316-333.

38. Barresi V, Grosso M, Barresi G. Immunohistochemical evidence of caveolin-1 expression in the human fetal and neonatal striated muscle and absence in the adult's. Appl Immunohistochem Mol Morphol. 2008;16:267-273.

39. Muller J, Vayssiere N, Royuela M et al. Comparative evolution of muscular dystrophy in diaphragm, gastrocnemius and masseter muscles from old male mdx mice. $J$ Muscle Res Cell Motil. 2001;22:133-139.

40. Stedman HH, Sweeney HL, Shrager JB et al. The mdx mouse diaphragm reproduces the degenerative changes of Duchenne muscular dystrophy. Nature. 1991;352:536-539. 
41. El-Yazbi AF, Cho WJ, Cena J, Schulz R, Daniel EE. Smooth muscle NOS, colocalized with caveolin-1, modulates contraction in mouse small intestine. $J$ Cell Mol Med. 2008;12:1404-1415.

42. Gomez-Ruiz A, Milagro FI, Campion J, Martinez JA, de Miguel C. Caveolin expression and activation in retroperitoneal and subcutaneous adipocytes: influence of a high-fat diet. J Cell Physiol. 2010;225:206-213.

43. Klagsbrun M, D'Amore PA. Vascular endothelial growth factor and its receptors. Cytokine Growth Factor Rev. 1996;7:259-270.
44. Saito T, Yamamoto Y, Matsumura T, Fujimura H, Shinno S. Serum levels of vascular endothelial growth factor elevated in patients with muscular dystrophy. Brain Dev. 2009;31:612-617.

45. Messina S, Mazzeo A, Bitto A et al. VEGF overexpression via adeno-associated virus gene transfer promotes skeletal muscle regeneration and enhances muscle function in $\mathrm{mdx}$ mice. Faseb J. 2007;21:3737-3746.

46. Sonveaux P, Martinive P, DeWever J et al. Caveolin-1 expression is critical for vascular endothelial growth factorinduced ischemic hindlimb collateralization and nitric oxide-mediated angiogenesis. Circ Res. 2004;95:154-161.

Submitted: 23 August, 2010 Accepted after reviews: 4 February, 2011 